19 Revue d'histoire du XIXe siècle

Société d'histoire de la révolution de 1848 et des

révolutions du XIXe siècle

$26 / 27 \mid 2003$

Varia

\title{
La " garde de la République » et le coup d'État du 2 décembre
}

Fabien Cardoni

(2) OpenEdition

Journals

Édition électronique

URL : https://journals.openedition.org/rh19/739

DOI : 10.4000/rh19.739

ISSN : $1777-5329$

Éditeur

La Société de 1848

Édition imprimée

Date de publication : 1 décembre 2003

Pagination : 111-130

ISSN : 1265-1354

Référence électronique

Fabien Cardoni, «La « garde de la République » et le coup d'État du 2 décembre », Revue d'histoire du XIXe siècle [En ligne], 26/27 | 2003, mis en ligne le 19 février 2008, consulté le 28 février 2022. URL: http://journals.openedition.org/rh19/739 ; DOI : https://doi.org/10.4000/rh19.739

Ce document a été généré automatiquement le 28 février 2022.

Tous droits réservés 


\title{
La « garde de la République » et le coup d'État du 2 décembre
}

\author{
Fabien Cardoni
}

«On arrivait à Mazas, un maréchal des logis de la
garde républicaine se trouvait dans le greffe, où,
en écrouant M. Charles Lagrange, on dut faire
l'inventaire des pièces à conviction rapportées de
chez lui : « Voici un sabre qui m'a appartenu,
s'écria ce sous-officier, nommé Kerkan; il doit
porter le $n^{\circ} 487$, et m'a été volé le 23 février 1848 ;
à la caserne de la garde municipale, dont j'étais
brigadier. On vérifia ; le fait était vrai » ${ }^{1}$.

1 Nombreuses sont les sources et les témoignages des contemporains, quelles que soient leurs opinions politiques, qui font état de la participation des gardes républicains à la répression de la résistance au coup d'État. Cependant l'historiographie de la garde républicaine et de la gendarmerie, y compris la plus récente ${ }^{2}$, n'évoque que très brièvement la participation de ce corps au coup de force de Louis-Napoléon. Ce constat invite à se pencher sur le degré réel d'implication de cette troupe, qui, dès ses débuts, a pour vocation d'être la " garde de la République ».

2 S'il est important de discerner les faits établis de la légende noire, il est nécessaire de préciser, dans un premier temps, l'état d'esprit du corps à la veille du 2 décembre. Comment en dépit de son recrutement hétérogène et des clivages qui la traversent, cette troupe a conservé sa cohésion lors de la répression ${ }^{3}$ ? Il convient donc de rappeler au préalable les conditions de sa formation et son évolution depuis la révolution de février. L'étude de son action exacte lors de la « révolution militaire » de 1851 permet d'analyser pourquoi sa participation est minorée et d'envisager la construction - et la perpétuation - de cette mémoire amputée. En d'autres termes, de quelle manière et dans quel but, les historiographes de la garde républicaine ont transfiguré cet acte en un non-événement pour le corps?

Une garde « républicaine » ?Une troupe hétérogène 
Une des premières mesures du Gouvernement provisoire, le 25 février 1848, est de dissoudre la garde municipale qui avait opposé une résistance aussi farouche que solitaire aux insurgés ${ }^{4}$. Des citoyens, républicains fervents et pour la plupart condamnés politiques de la Monarchie de Juillet, constituent alors des groupes armés qui s'auto-attribuent la garde de divers bâtiments officiels de la capitale dont l'Hôtel de Ville et la Préfecture de police. Décidés à rester en armes pour assurer la police de la ville, ces groupes indisciplinés exercent également une pression directe sur le Gouvernement provisoire. Le nouveau pouvoir va chercher à les unifier afin d'en prendre le contrôle. Ainsi, le décret du 24 avril 1848 prévoit la réunion en une «garde civique ", de la garde républicaine de l'Hôtel de Ville et du corps des Lyonnais. Les Montagnards, sous l'autorité directe du préfet de police, Caussidière, sont tenus à l'écart. Lors de la journée du 15 mai 1848, on accuse cette "garde civique ", forte de plus de 2000 hommes, et surtout les Montagnards, d'avoir sinon favorisé le développement de l'émeute, du moins de ne pas avoir essayé de l'endiguer. Le lendemain du 15 mai, la commission du pouvoir exécutif dissout tous ces groupes et constitue une "garde républicaine de Paris ", initiant par-là une première épuration des éléments les plus radicaux. Lors des journées de Juin, une faible partie de ces gardes républicains ont combattu aux côtés de l'émeute. Nombre d'entre eux sont tués ou condamnés. Afin de remplacer ces effectifs perdus et de modifier la physionomie du corps, on autorise, à partir de juillet 1848 , le retour des ex-gardes municipaux licenciés. Le pouvoir y injecte également des militaires de la ligne et des gendarmes départementaux. La garde républicaine, à l'image de la République, subit un tournant conservateur. La gardienne de la République reflète la nature du régime.

En 1849, cet ensemble hétéroclite de citoyens armés n'offre toujours pas, aux yeux de la Commission exécutive, toutes les garanties de fiabilité. Il est alors décidé, par le décret du $1^{\text {er }}$ février 1849, de placer la garde républicaine dans les attributions du ministère de la Guerre et de la rattacher à la gendarmerie. Une nouvelle purge commence. Cette disposition exclut en effet tous les gardes qui ne remplissent pas les conditions (taille minimale, services militaires antérieurs...) pour devenir gendarme et favorise, là encore, le retour de nombreux ex-gardes municipaux. En janvier 1852, on compte plus de quatre cents anciens gardes municipaux sur un total d'environ 2100 gardes républicains ${ }^{5}$. Cette quadruple source de recrutement (ex-révolutionnaires, militaires, gendarmes des départements, gardes municipaux) explique les doutes qui planent sur la solidité de cette troupe enrégimentée.

5 Un autre élément contribue à expliquer que les bonapartistes aient pu douter de la fiabilité de cette troupe. En effet, l'ensemble des officiers a été choisi plus ou moins directement par Changarnier ${ }^{6}$ car il a présidé la commission de réorganisation de la garde républicaine. À propos du chef de corps, Gastu, le préfet de police Maupas écrit dans ses Mémoires : « le colonel, brave militaire sans doute, était l'une des créatures du général Changarnier. Je m'étais assuré, tout récemment, que ses rapports continuaient avec le général. Je ne pouvais donc me fier à lui que dans une mesure limitée. $\mathrm{Ne}$ pouvant, en aucun cas, risquer ma confiance, je pris le parti de me passer de son concours " ${ }^{7}$. C'est bien Changarnier qui l'a proposé pour occuper ce commandement. Il dit de lui dans une lettre de proposition pour la croix d'officier de la légion d'honneur : "Tout ce que je me sentais en droit d'attendre de dévouement, d'effort de la part d'un homme laborieux, intelligent et ferme comme lui, M. le colonel Gastu l'a consacré à l'œuvre de reconstitution qui lui était confié. En moins de six mois, il a obtenu des 
résultats, dont le Président de la République et vous avez pu vous faire une idée dans votre visite récente des casernes. D'un corps mal administré, mal discipliné, d'un corps dont le nom seul inspirait la méfiance à la partie saine de la population de Paris, il a fait une troupe avec laquelle les ennemis de l'ordre auront désormais à compter ${ }^{8}$. Ainsi, il n'est pas remplacé dans les mois qui précédent le coup d'État. Toutes les appréciations contenues dans les revues individuelles d'inspection de son dossier administratif sont élogieuses. Sa nomination au poste de colonel de la garde républicaine, le 19 juillet 1849 , semble alors tout à fait justifiée.

6 L'hétérogénéité de son recrutement contribue à diversifier les sensibilités politiques au sein de la garde. Le corps devient une sorte de "République en miniature ".

$7 \quad$ Afin d'apprécier les tensions internes au sein de cette troupe au cours de ses premières années d'existence, il est important d'évoquer au préalable le sort d'ex-gardes républicains qui ont participé à la résistance au coup d'État.

8 Paul Belouino rapporte que «rue Rambuteau [...] Il y avait là, parmi les insurgés, d'anciens satellites de Caussidière, faisant admirablement le coup de feu " ${ }^{9}$. Par ailleurs, la présence d'«anciens officiers de la garde républicaine " à la réunion du comité de résistance, le 3 décembre, rue Popincourt, est remarquée par deux témoins ${ }^{10}$. Un ancien garde semble avoir été fusillé par des gardes républicains, le 5 ou le 6 décembre vers deux heures de l'après-midi sur le pont Saint-Michel, car il portait « des munitions de guerre et des poignards $"{ }^{11}$.

On a pu retrouver la trace de treize ex-gardes républicains parmi les quelques 26000 inculpés par les commissions mixtes ${ }^{12}$. Il est néanmoins difficile de repérer ces anciens gardes républicains soit parce qu'ils n'ont pas été référencés comme tels, soit encore qu'ils sont morts lors des combats, soit enfin qu'ils n'ont pas été arrêtés. Ces individus sont signalés comme " ex-garde républicain » ou « ayant appartenu aux Montagnards » dans la rubrique profession ou dans la colonne réservée aux antécédents judiciaires et aux observations. Cette indication apparaît parfois comme un motif suffisant de condamnation et constitue, dans tous les cas, une condition aggravante au même titre que d'avoir participé à l'insurrection de juin 1848 ou de faire - ou d'avoir fait - partie d'une société secrète. En effet, cinq individus sont condamnés sans que leurs participations soient démontrées. Deux sont presque aussitôt remis en liberté, trois autres sont condamnés à l'appui de preuves incontestables. De ces treize individus, on ne connaît le destin que de dix d'entre eux ${ }^{13}$. Les dossiers de condamnations par les commissions mixtes conservés au Service historique de l'armée de Terre ainsi que les quelques dossiers de demande de grâce nous apportent de nombreuses indications non seulement sur les conditions des arrestations et des enquêtes mais aussi sur la garde républicaine d'avant décembre 1851.

Lisons par exemple, le rapport de Marquisan, capitaine adjudant-major de la garde républicaine, concernant l'arrestation de Bourdenet, ex-brigadier du même corps : «Le 4 décembre dernier, vers 3 heures de l'après-midi, quelques instants après que des coups de feu s'étaient fait entendre sur le quai de Gèvres, je me trouvais sur le quai de l'Horloge ; le brigadier Holchon que je rencontrai devant la Conciergerie me dit en me montrant un individu : "Voilà Bourdenet, j'avais presque envie de l'arrêter parce qu'il veut traverser le pont et qu'il pourrait bien aller aux barricades". J'appelai Bourdenet et lui demandai ce qu'il faisait là dans un pareil moment, il me répondit qu'il rentrait à son domicile. Comme ce brigadier avait manifesté quelque mécontentement à son départ du corps, je crus, sans écouter ses protestations, devoir l'arrêter par mesure de 
précaution [souligné dans le document] et je le fis mettre dans les rangs de la compagnie du capitaine d'Eggs, pour y être surveillé » ${ }^{14}$.

11 La présence de Luneau en uniforme de lieutenant de l'ancienne garde républicaine derrière une barricade, est évoquée dans de nombreux témoignages ${ }^{15}$. Pour les opposants au coup, c'est bien sûr le symbole de la République qui se défend. Pour les pro-bonapartistes, il personnifie le caractère résolument révolutionnaire de l'ancienne garde républicaine. Son dossier de condamnation par contumace nous apprend que, le 4 décembre 1851, il est monté chez lui pour revêtir cet uniforme, lui conférant une autorité sur les combattants des barricades. Il est avéré qu'il dirigea la résistance à la barricade de la rue Saint-Martin. La déposition du 12 janvier 1852 de Julien Thuillier, restaurateur de la rue du faubourg Saint-Martin, indique : «J'ai vu arriver par la rue du Ponceau un homme à la longue barbe revêtu de l'uniforme de l'ancienne garde républicaine, il avait l'épée à la main et haranguait une cinquantaine de personnes qui l'entouraient, il criait Vive la République et ajoutait que la garde républicaine était avec eux et s'est ensuite dirigé du côté du faubourg Saint-Martin où il a encore harangué le peuple ${ }^{16}$. Ces mots de Luneau, s'ils sont véridiques car Thuillier semble confondre deux personnes, visent évidemment à stimuler les combattants mais peut-être sont-ils aussi le reflet d'un espoir sincère en un ralliement à l'insurrection de toute ou une partie de la garde républicaine. Cet espoir est-il fondé ? En dépit de toutes les épurations à peine déguisées (26 mars, 16 mai, juin 1848, $1^{\text {er }}$ février 1849), des combattants de février sont toujours en service dans la garde républicaine début décembre $1851{ }^{17}$. Par ailleurs, il est établi que des ex-gardes républicains conservent des relations à la Préfecture de police. Par exemple, le directeur du dépôt, Cabuchet, connaît bien l'ancien lieutenant-colonel Caillaud qui est reconnu par des sergents de ville et arrêté le 4 décembre sur le quai de l'École ${ }^{18}$ ainsi que Boiteux qui se rend le samedi 6 décembre à la Préfecture pour y " voir des connaissances » ${ }^{19}$. Enfin, on peut supposer que certains anciens gardes républicains maintiennent des relations avec des gardes encore en activité.

Un rapport du général de la Rüe, inspecteur du corps, en 1853, laisse, lui aussi, percevoir des divergences profondes, voire des antagonismes, au sein de cette troupe. "On voit d'après ce relevé sommaire combien d'éléments différents participent à la composition de la garde de Paris, plusieurs de ces éléments sont de nature à se bien concilier, mais il n'est pas douteux que celui qui prend sa source à l'ancienne gendarmerie régionale et surtout dans les Montagnards, ne doive encore offrir aujourd'hui des causes de désunion et d'indiscipline, nécessairement, les anciens gendarmes mobiles doivent voir avec peine dans leurs rangs et surtout doivent obéir avec répugnance à des hommes contre lesquels ils ont si longtemps et si honorablement combattu et leurs antipathies à cet égard doivent être souvent partagées par ceux de leurs camarades qui viennent de la gendarmerie et de l'Armée $"{ }^{20}$. Les remarques de De la Rüe en 1855 s'appliquent a priori à la composition de la troupe en 1851 et en effet les rapports d'inspection du corps - du général Laverderie pour l'année 1850 ou encore du général Carrelet (ancien commandant de la garde municipale) pour 1851 -, font déjà état de dissensions internes et appellent à s'inspirer des valeurs de cohésion et de fidélité de l'ancienne garde municipale ${ }^{21}$. Cependant ces inspecteurs généraux n'hésitent pas à affirmer que l'on peut compter sur cette troupe en cas de troubles ${ }^{22}$. Une anecdote confirme cette appréciation: « Le lendemain du jour où fut adoptée la proposition [Baze], un des questeurs rencontra un 
de ses meilleurs amis, M. de Prémonville, chef d'escadron dans la garde de Paris et lui dit :

- Eh bien, si je te donne un ordre maintenant, que me répondras-tu?

- Pardieu, je te répondrai : pour que je t'obéisse, va prendre d'abord ta pelure (ton uniforme) ${ }^{23}$.

13 Il n'est guère étonnant que la fidélité à la hiérarchie, le respect de la légalité et l'obéissance au pouvoir politique quel qu'il soit, priment dans l'attitude des cadres de la garde. Mais qu'en est-il pour les gardes? Désunis, se retrouvent-ils autour de la personne de Louis-Napoléon?

Louis-Napoléon et la garde républicaine

14 Sans pouvoir apprécier précisément le poids respectif des différentes tendances politiques dans la garde, ni même le degré de politisation de ces militaires, il est néanmoins intéressant d'interroger leur attitude, dans les mois qui précèdent le coup d'État, vis-à-vis notamment de la personne et de la politique du Prince-Président.

Afin de tenter de répondre à cette question, seules les actions favorables visant nominativement la garde républicaine sont à prendre en considération. Tout d'abord, la conséquente augmentation de solde pour tous les grades au mois d'août $1849^{24}$ est sans aucun doute unanimement appréciée. De plus, l'ordonnance du 27 octobre 1849, portant modification du cadre de cette troupe, permet de rendre aux sous-officiers les avantages qui étaient assurés à ceux de la garde municipale. Ils ont désormais droit à la moitié des sous-lieutenances d'infanterie et de cavalerie offerts dans la garde républicaine. Enfin, la loi du 18 juin 1851 accorde le traitement de légionnaires aux officiers, sous-officiers et soldats de la garde décorés pour leur conduite lors des journées de juin. Il s'agit là d'une récompense officielle et pécuniaire pour leur combat contre les radicaux, contre une certaine République. Il est possible que le PrincePrésident ait initié le rattachement de la garde républicaine à la gendarmerie. Il est probable, en revanche, que cette mesure soit souhaitée par les militaires du corps et tout particulièrement par les ex-gardes municipaux.

16 L'entreprise bonapartiste de séduction de ces militaires se manifeste plus explicitement encore, lors des apparitions publiques de Louis-Napoléon. Ainsi, le 13 février 1850, il passe en revue les diverses casernes occupées par la garde républicaine. Lors de cette revue, il décerne «six décorations de la Légion d'honneur [...] dont une pour un simple gendarme, blessé en Juin » ${ }^{25}$. En août 1850, Louis-Napoléon passe une nouvelle revue des unités de la gendarmerie parisienne, de la gendarmerie mobile et de la garde républicaine. Après la distribution de nouvelles croix et le défilé, tous les officiers et les sous-officiers des deux corps sont conviés à un banquet à l'Élysée. Le Journal de la gendarmerie écrit qu'« à huit heures et demie, à l'exemple du Président tout le monde s'est levé et le cri de vive Napoléon a retenti d'un bout à l'autre du jardin. Après quelques instants de promenade, employés par le Président à s'entretenir avec les officiers et sous-officiers et à recevoir les témoignages de sympathie et de dévouement que ces braves militaires lui prodiguaient à l'envi, Louis-Napoléon est rentré dans ses appartements et les invités se sont retirés, le cœur plein de reconnaissance, et vivement touchés de l'accueil si bienveillant et si cordial de l'hôte illustre qui les avait conviés à cette fête de famille " ${ }^{26}$. L'auteur, le chef d'escadron en retraite Cochet de Savigny, n'est pas connu pour son impartialité. Mais ces démonstrations sont sans doute sincères. Le 12 novembre 1851 a lieu une revue de la brigade de réserve. Elle fournit une nouvelle occasion au Président de décorer, parmi d'autres militaires, deux 
officiers, trois maréchaux des logis et un garde à cheval ${ }^{27}$. Les marques de bienveillance et les mesures favorables dont sont l'objet ces militaires n'ont pu les laisser indifférents. Louis-Napoléon par ses attentions répétées aux militaires construit peu à peu une relation paternelle et une "alliance sentimentale » avec l'armée. Ses deux aspects seront déterminants dans la réussite du coup d'État. Le Président de la République se pose en restaurateur du prestige et de l'honneur de l'armée atteintes lors de la révolution de février. Ce discours touche particulièrement les vieux militaires et les ex-gardes municipaux.

17 Bonaparte dispose-t-il d'agents dans la garde républicaine avant même son élection à la présidence? Un ancien garde, Louis Christ Boiteux, écrit «que s'il perdit sa place de cantinier [dans la garde républicaine] c'est en raison de ce qu'il fut reconnu pour avoir distribué dans différentes casernes des bulletins de vote pour la nomination du Prince Louis-Napoléon, à la présidence ${ }^{28}$. Il convient de prendre avec circonspection ce témoignage de 1852, devant la commission mixte. Cette déclaration émane d'un individu qui cherche avant tout à atténuer les accusations portées contre lui. Un document laisse entendre en revanche qu'il réside dans la troupe des sympathisants de la République radicale : «Un nommé Huault Frédéric, maréchal des logis chef dans la garde républicaine vient de passer quelques jours à Saumur, pendant tout le temps de son séjour ici il a continuellement été en rapport avec les hommes les plus avancés dans le parti rouge. L'un de nos agents de police qui surveillait ses mouvements l'a vu, selon son expression, en grande conversation avec un nommé Gustave Swamberg, architecte, signalé depuis longtemps comme l'un des hommes les plus dangereux par la propagande socialiste qu'il exerce sur nos ouvriers. Ce Gustave est d'origine étrangère, et étranger lui-même à Saumur. Il ne peut donc y avoir entre lui et Huault, qui luimême n'est pas de Saumur, d'autres rapports que ceux de la politique ${ }^{29}$. La surveillance politique qui se généralise n'épargne pas la garde républicaine.

Par ailleurs, il est illusoire de tenter d'évaluer sérieusement les résultats de la propagande bonapartiste sur les gardes républicains en examinant les chiffres du plébiscite de décembre 1851. En effet, la garde républicaine vote le 3 ou le 4 décembre conformément à l'ordre de Saint-Arnaud du 2 décembre. Les gardes votent donc sur des registres, alors que des combats ont encore lieu. Le 4, Louis-Napoléon rétablit le vote par bulletins secrets et les registres sont détruits mais on ne procède pas à une deuxième consultation. Le résultat du plébiscite est de 1983 oui pour autant de votants ${ }^{30}$. On connaît notamment par le témoignage de Victor Frond, sous-lieutenant à la $4^{\mathrm{e}}$ compagnie du bataillon de sapeurs-pompiers de Paris, toute l'étendue des pressions exercées par «les officiers supérieurs [qui] extorquaient des votes d'approbation à de malheureux soldats qu'on trompait systématiquement et par quels procédés d'intimidation on forçait ceux qui avaient voté non à se rétracter " ${ }^{31}$. Malgré le procédé du vote public et les pressions hiérarchiques, il y eu des nons dans l'armée de Paris ${ }^{32}$. On ne peut donc exclure que les révolutionnaires de février encore présents dans la garde républicaine et les anciens gardes municipaux ont voté massivement, mais pour des raisons différentes, pour la conservation de Louis-Napoléon Bonaparte. Pour les premiers, il est le réel défenseur de l'esprit de la République de février et le restaurateur du suffrage universel. Les seconds le considèrent, quant à eux, avant tout comme le protecteur de l'armée et l'incarnation de la revanche. 


\section{L'examen de son rôle lors des événements de décembre 1851 permet d'y répondre en} partie.

L'action contrastée de la garde républicaineLa position de la garde républicaine dans le dispositif et les préparatifs du coup d'État

Sans entrer dans le détail des tactiques de la guerre des rues, le rôle de la garde républicaine en cas de troubles est périlleux. Si par sa dispersion dans le cœur de la capitale, elle est capable de contenir rapidement un début d'agitation localisée, elle peut être, du fait même de cette dispersion, très facilement débordée par une insurrection. Conscient de cette faiblesse, le commandement militaire ${ }^{33}$, tirant les leçons de la révolution de février et s'inspirant du plan Gérard, du nom d'un maréchal ministre de la Guerre sous la Monarchie de Juillet, va pendant la Seconde République prévoir de l'employer en unités regroupées aux côtés des autres troupes de la garnison de Paris. On trouve ainsi dans les archives de l'armée de Terre un plan détaillant les positions de toutes ces troupes en cas de guerre des rues ${ }^{34}$. Ce plan est plusieurs fois remanié y compris après le 2 décembre ${ }^{35}$. Il est admis que la version de la fin de l'année 1851 a été préparée par le préfet de police Carlier ${ }^{36}$. Il place la garde républicaine au sein de la $4^{\mathrm{e}}$ brigade de réserve de la $1^{\text {ère }}$ division du général et prévoit que les deux bataillons d'infanterie de la garde restent aux ordres du préfet de police, c'est-à-dire aux abords de la Préfecture de police. La moitié du $2^{\mathrm{e}}$ escadron, établie rue de Tournon près du Luxembourg, est à la disposition du général Renault alors que l'autre moitié du $2^{\mathrm{e}}$ escadron et le $1^{\mathrm{er}}$ escadron sont à l'Hôtel de Ville aux ordres du général Levasseur. La garde républicaine est destinée à être conservée comme une troupe de réserve.

Contrairement aux dispositions de combat prédéfinies, la quasi-totalité de l'infanterie et de la cavalerie de la garde républicaine est concentrée à la Préfecture de police, à l'entière disposition du préfet ${ }^{37}$. Elle a pour mission principale de défendre l'île de la Cité et plus particulièrement la Préfecture. Lors d'une révolution, la Préfecture de police est un objectif stratégique évident par sa situation dans Paris et par les renseignements qu'elle contient. La Préfecture est également un symbole politique à faire tomber. La garde reste ainsi confinée jour et nuit en bivouac dans la cour de la Préfecture jusqu'au 8 décembre ${ }^{38}$. Si cette position est logique, elle n'est pas prévue. Est-ce le signe d'une certaine défiance du préfet de police vis-à-vis de la garde ? On est en droit d'en douter car si cette méfiance était réelle, il y aurait un danger certain à la maintenir à la Préfecture. Il est en revanche beaucoup plus probable que Maupas souhaitent conserver sous la main une troupe composée de nombreux éléments - exgardes municipaux, combattants de février et de juin - expérimentés dans la guerre des rues. Comme le prévoit les textes, la garde est sous l'autorité directe du préfet de police pour son emploi. Il peut ainsi aisément se passer du colonel Gastu. Enfin, Maupas se plaint, dès le 2 décembre, de l'insuffisance des forces dont il dispose. Ce changement par rapport au plan initial est donc très probablement le résultat des demandes pressantes du préfet de police au commandant de l'armée de Paris. On peut donc penser que Maupas, bien que conscient des clivages au sein de la garde, s'est vu contraint de lui faire confiance, car il ne possède pas d'autre troupe pour le protéger et avec lui, la Préfecture de police.

Seul Maupas nous renseigne sur les préparatifs du coup : «De temps en temps, je faisais doubler les postes de la Préfecture, et plusieurs fois depuis quelques jours, j'avais fait stationner dans les cours un piquet d'hommes à pied et un autre d'hommes à cheval de 
la garde municipale. Leur mission annoncée était de se porter au besoin sur les points où je faisais opérer d'importantes arrestations de réfugiés de Londres et de chefs de sociétés secrètes. Le $1^{\text {er }}$ décembre, je donnai l'ordre de nouveau et, à trois heures du matin, je mandai directement, dans mon cabinet, plusieurs capitaines que je savais disposés à m'obéir sans hésitation. Je leur donnai mes instructions, et, à l'heure dite, ils étaient, à la tête de leurs soldats, au poste que je leur avais assigné » ${ }^{39}$. S'il n'existe pas dans les archives d'état détaillé de l'action de la garde républicaine à l'instar des deux bataillons de gendarmerie mobile ${ }^{40}$, nous disposons, en revanche, d'un rapport du colonel Gastu sur l'action de sa troupe ${ }^{41}$. En le confrontant à d'autres documents officiels, aux récits de témoins et aux mémoires de contemporains ${ }^{42}$, il est possible de déterminer avec précision les actions des gardes républicains au cours de chacune des journées parisiennes du coup d'État.

Sans coup férir

Le 2 décembre, « dès 6 heures du matin [...], sur la réquisition de M. le préfet de police, je mis à la disposition de ses commissaires un demi bataillon qui fut subdivisé en quatorze détachements, pour opérer des arrestations. [...] Quelques instants après, je fis venir, toujours d'après les ordres du préfet de police, à la disposition duquel le corps avait été placé, toute la cavalerie sur les positions de combat. Dans le courant de cette journée, le corps n'a eu qu'à obtempérer aux différentes réquisitions de $\mathrm{M}$. le préfet ou des agents de son administration pour des perquisitions et des arrestations ${ }^{43}$. Le capitaine Baudinet, commandant un détachement de gardes ${ }^{44}$, fut ainsi adjoint au commissaire Lerat pour arrêter Changarnier à son domicile. Ces militaires l'escortent jusqu'à la prison Mazas comme ils le font pour d'autres représentants arrêtés ${ }^{45}$. Des détachements de gardes sont également employés à l'occupation de journaux interdits. À ce sujet, Hugo raconte : "Serrière imprimait La Presse et l'Avènement du Peuple [...]. Le 2, à sept heures du matin, l'imprimerie avait été envahie par vingt-huit soldats de [la] garde républicaine, que commandait un lieutenant nommé Pape (décoré depuis pour cela). [...] Les ouvriers avaient résisté; un margeur avait dit aux soldats: Nous imprimerons malgré vous. Alors étaient arrivés quarante nouveaux gardes municipaux, avec deux maréchaux des logis et quatre brigadiers, et, tambour en tête, un détachement de ligne commandée par un capitaine. Girardin survint, indigné, et protesta avec tant d'énergie qu'un maréchal des logis lui dit: Je voudrais avoir un colonel comme vous. Le courage de Girardin gagna les ouvriers, et à force d'adresse et d'audace, sous l'œil même des gendarmes, ils parvinrent à imprimer les proclamations de Girardin à la presse à bras, et les nôtres à la brosse. Ils les emportaient, tout humides, et par petits paquets, sous leurs gilets. Heureusement on était ivre. Les gendarmes faisaient boire les soldats, les ouvriers profitaient de cette gaîté pour travailler. Les gardes municipaux riaient, juraient, faisaient des calembours, buvaient du vin de Champagne et du café $"{ }^{46}$. Le témoignage d'Ernest Caylus ${ }^{47}$ confirme l'attitude des gardes: "Je retourne au National vers 1 heure. Il parait que les gardes républicains composant la compagnie qui occupait la maison n'étaient pas assez stricts dans leurs rapports avec les personnes nombreuses qui venaient chercher des nouvelles, car on les remplaça par une compagnie d'infanterie de ligne $"{ }^{48}$. Si Caylus dit vrai, doit-on voir dans ces imprimeries qui continuent de fonctionner malgré l'occupation militaire, un laxisme étonnant ou la preuve de l'existence de gardes favorables au développement de la résistance ? En l'absence d'éléments supplémentaires - Caylus et par conséquent Hugo sont les seuls à nous rapporter ces faits -, il est impossible de trancher. Les presses de La Patrie, de La République, du Siècle, et même du Moniteur parisien, favorable à 
Bonaparte, soit au total treize imprimeries, sont occupées de la sorte. Dans la nuit du 3 au 4, vers trois heures du matin, elles sont toutes évacuées ${ }^{49}$. Durant cette même journée du 2 décembre, une compagnie intervient pour dissoudre sans grande opposition la réunion de la Haute Cour de justice ${ }^{50}$. On trouve également des gardes en nombre dans la cour ${ }^{51}$ et au greffe de la prison Mazas ${ }^{52}$. Des gardes à cheval refoulent, place Saint-Sulpice, un rassemblement de jeunes gens qui se dirigeaient vers la réunion des représentants à la mairie du $\mathrm{X}^{\mathrm{e}}$ arrondissement ${ }^{53}$. Le reste de la garde républicaine se partage avec un bataillon du $6^{\mathrm{e}}$ léger et une demie batterie d'artillerie, la défense de la Cité ${ }^{54}$. Ils sont répartis sur le quai aux Fleurs, la place Dauphine, le Pont-Neuf et la cour de la Préfecture de police avec pour mission à la fois de repousser une attaque sur le Palais de justice ou sur la Préfecture de police et de contrôler l'accès aux ponts afin d'empêcher les communications entre les deux rives. Gastu nous explique: «Je fis mettre une pièce dans la cour de la Sainte-Chapelle pour l'avoir plus à portée de la place du Palais de Justice et des ponts au Change et Saint-Michel, points sur lesquels elle pouvait être utilement employée. Les deux autres restèrent en position sur la Place Dauphine ${ }^{55}$. L'île de la Cité prend ainsi l'aspect d'une forteresse solidement défendue. Dans la soirée, la cavalerie de la garde républicaine est renvoyée dans ses casernes ainsi que deux compagnies du $6^{\text {ème }}$ léger et la demie batterie d'artillerie ${ }^{56}$.

Le 3 décembre dans la matinée, Maupas envoie, en reconnaissance, un officier de la garde au faubourg Saint-Antoine. C'est par lui qu'il apprend la mort de Baudin ${ }^{57}$. Vers midi, un détachement de gardes qui emmène des prisonniers est attaqué sur le boulevard Beaumarchais par la foule ${ }^{58}$. Des gardes de la caserne des Minimes sortent à son secours. Un bref mais vif combat s'engage. Un seul individu est arrêté ${ }^{59}$. Peu avant 5 heures de l'après-midi, deux barricades dans la rue Saint-Martin sont facilement détruites par un détachement de gardes républicains. "Les défenseurs de ces barricades n'étaient pas armés ${ }^{60}$. Dans cette même journée, un détachement de la garde républicaine dirigé par le commissaire de police Boudrot participe aux arrestations de journalistes au divan de la rue Lepelletier ${ }^{61}$.

Hugo nous raconte comment un ancien capitaine de la $8^{\mathrm{e}}$ légion de la garde nationale en 1848, nommé Jourdan, se propose le matin du 4 décembre de faire un coup de main sur l'Hôtel de Ville et la Préfecture de police ${ }^{62}$. Maupas en est informé car Gastu écrit que: "d'après les renseignements parvenus à $\mathrm{M}$. le préfet de police " ${ }^{63}$, il doit s'attendre à une attaque vers midi. Le " plan favori des insurgés, plan qui consistait à occuper la Cité, à délivrer leurs prisonniers et à se fortifier dans l'île " ${ }^{64}$ est mis à exécution. «Le rassemblement du quai aux fleurs [...] se ruait sur le pont Saint-Michel, en forçait le passage et se précipitait, au pas de course, dans la rue de la Barillerie, à l'entrée principale de la Préfecture de police ${ }^{65}$. Les assaillants ont profité d'une diversion. En effet vers deux heures de l'après-midi, «plusieurs coups de feu venaient d'être tirés de quelques maisons des rues de la Cité, de Constantine et du bas de la rue Saint-Jacques. [...] une compagnie fut envoyée au-delà du Petit-Pont, où déjà les émeutiers avaient placé une grande charrette en travers de la rue Saint-Jacques. [...] Au même moment des coups de feu partirent des quais de Gesvres et Pelletier, bien que le colonel eût déjà fait occuper par des gardes les maisons d'angles de la rue PlancheMibray ${ }^{66}$. La vigueur de la riposte des gardes met en déroute les insurgés au bout d'une heure. Ce combat fait au moins une victime du côté des assaillants ${ }^{67}$ et deux blessés parmi les gardes ${ }^{68}$. La troupe procède à de nombreuses arrestations ${ }^{69}$. 

fausses nouvelles, et qui furent passés par les armes et lancés dans la Seine " ${ }^{73}$. S'il est, à encore, impossible de déterminer le nombre exact des mises à mort d'individus pris armés ou les mains noires de poudre - dont l'ex-garde républicain évoqué plus haut - , elles sont plausibles, car conformes aux ordres du ministre de la Guerre. L'histoire du jardinier à Passy ${ }^{74}$, Joseph Berger, 43 ans, qui tente de traverser le Pont-Neuf dans la nuit du 4 au 5 décembre dissimulant une carabine sous sa blouse, est avérée. Il est arrêté par les gardes qui surveillent le pont et «passé par les armes » ${ }^{75}$. Blessé par balle à la cuisse, il est jeté à la Seine. Il survit cependant à sa blessure et à sa chute. On raconte aussi comment un garde a écrasé la tête d'un mourant ${ }^{76}$, comment d'autres assommaient les prisonniers tout juste arrivés à la Préfecture de police «à coups de massue à boules de plomb ${ }^{77}$. Ces affirmations sont difficilement vérifiables. La cruauté et la brutalité font partie des thématiques anciennement associées aux gardes municipaux. Par ailleurs, les gardes républicains - à l'instar des autres soldats - sont dépeints par les détracteurs du coup d'État comme ivres de vin tout au long des journées parisiennes ${ }^{78}$. La lutte se déplace sur le terrain des représentations de l'événement.

De l'événement au non-événementLes rapports officiels et la presse corporative : l'« histoire » immédiate

8 La sion proposée par Maupas dans son Rapport sur les événements de décembre - il en est de même dans ses Mémoires - a deux buts : mettre en avant son action et prouver le péril de sa situation à la Préfecture de police. Son récit de l'attaque de l'île de la Cité laisse croire à un combat d'une grande ampleur et il en fait un des événements majeurs de l'insurrection parisienne. Il amplifie par conséquent le rôle de la garde républicaine. A l'issue du combat, le préfet adresse des félicitations ${ }^{79}$ à la troupe. Il loue "l'intrépidité", «le dévouement» et "l'énergique attitude " ${ }^{80}$ de la garde. Ces compliments sont autant de remerciements d'un homme qui a douté de la fidélité de la garde républicaine, mais surtout qui a eu peur pour sa personne. Eugène Tenot s'en moque : «Les vingt ou trente jeunes gens qui mettaient ainsi la Préfecture en alarmes par quelques coups de feu, tirés presque hors de portée, [...] ont raconté depuis combien fut grande leur surprise lorsque, après plusieurs années, les dépêches [de Maupas] [...], révélées par M. le docteur Véron, leur apprirent l'effet produit par leur diversion ${ }^{81}$. Signe du caractère anecdotique de cet épisode, le général Magnan, commandant de la $1^{\text {ère }}$ division militaire, n'évoque pas ce combat dans son rapport au ministre de la Guerre ${ }^{82}$. Il ne cite, du reste, à aucun moment l'action des gardes alors qu'il salue l'action, plus visible, de la gendarmerie mobile.

29 À l'inverse de Maupas, le colonel Gastu avoue volontiers que «pendant la durée des événements, la garde républicaine n'a pas eu de combat à livrer. Son rôle s'est borné à garder les positions qui lui avaient été assignées et que l'émeute n'est pas venue attaquer. Dans les différentes missions qui leur ont été confiées par le préfet de police, les détachements du corps ont fait preuve d'énergie et de résolution; plusieurs officiers ont été chargés de missions particulières, ils s'en sont tous acquittés d'une manière 
digne d'éloges ${ }^{83}$. Le fait qu'il n'y ait pas à notre connaissance dans les archives de la correspondance de la Guerre, de rapport similaire de commandant d'unité, laisse penser que le rapport de Gastu répond à une demande motivée de l'autorité militaire. Le colonel cherche donc tout de même à défendre l'action de la garde républicaine et à justifier une action que l'on a considéré comme timide. L'annotation, en marge de ce rapport ${ }^{84}$, probablement du général Dulac, semble confirmer cette hypothèse.

À l'écart des principaux théâtres d'opération, dispersée ou cantonnée dans des tâches peu glorieuses et discrètes, la garde a du mal à faire valoir sa participation à la répression. Un moyen de savoir si son rôle est considéré comme négligeable par les autorités, est d'étudier les récompenses et en particulier le nombre des décorations attribuées aux gardes au lendemain du coup d'État par rapport aux militaires des autres corps de troupe. Mais à ce sujet, Louis Saurel remarque avec raison qu'« en réalité ce fut le pourcentage des décorés que l'on augmenta et, de cette augmentation, profitèrent des militaires proposés depuis quelques années ${ }^{85}$. En effet les décorations des gardes républicains sont peu en rapport avec les services rendus en décembre. Gastu écrit : « les propositions de récompense que j'ai l'honneur de vous soumettre, [...] je les ai toutes prises sur celles faites par $M$. l'inspecteur général à la dernière inspection " ${ }^{86}$. Ainsi, "MM. de Fenin et Pape, et les sieurs Vibratte et Biérard, qui avaient d'ailleurs été proposés pour la décoration à l'inspection générale, ont été nommés chevaliers dans l'ordre de la Légion d'honneur par décret du 13 janvier $1852 »^{87}$. De nombreux gardes sont également récompensés pour des blessures reçues pendant la révolution de février ${ }^{88}$. La garde républicaine est pour cette raison autant " décorée » que la gendarmerie mobile. Enfin, le décret qui ouvre un crédit pour indemniser ceux qui ont souffert de dommages matériels lors des événements de février et juin $1848^{89}$, dont notamment les militaires de l'ancienne garde municipale, a peut-être été perçu comme une récompense de l'action de la garde lors du coup d'État. Mais ce décret n'a pas de relation directe avec le 2 décembre dans la mesure où il n'est que la mise en œuvre d'une décision prise par la Seconde République et qu'il permet l'indemnisation de toutes les personnes, civiles ou militaires, touchées par les événements de 1848.

31 Cochet de Savigny dans sa Notice historique sur la révolution du mois de décembre $1851{ }^{90}$, dit, de la garde républicaine, que «si elle n'a pas eu à combattre, c'est que les dispositions prises avec les chefs, l'attitude résolue de la troupe, la promptitude et la vigueur avec lesquelles les tentatives de désordre, qui se sont manifestées sur quelques points de la Cité ou dans les environs, avaient été prévenues ou réprimées, ont fait comprendre aux hommes du désordre que tous leurs efforts seraient inutiles ${ }^{91}$. Selon lui, la garde républicaine fut un épouvantail efficace. Il évoque également la modeste intervention à la barricade de la rue Saint-Martin. Mais Cochet ne relate absolument pas la dispersion de la Haute Cour, les arrestations, la garde des prisonniers ou encore leurs transfèrements. Il occulte ainsi le caractère policier de l'action de la garde républicaine. Il ajoute: «Les bataillons de gendarmerie mobile, qui font partie d'une des brigades de l'armée de Paris, ont été engagés comme corps de troupe et ont eut conséquemment plus l'occasion de se faire remarquer que la gendarmerie de la Seine et que la garde républicaine ${ }^{92}$. Jugement pertinent car ce n'est qu'en fonction de cette visibilité que l'on peut expliquer la construction de cette mémoire. Mettre en relief uniquement des actions d'éclat qui sont peu nombreuses et surtout éluder les missions 
d'auxiliaire de police, a pour conséquence de minorer la participation de la garde au coup d'État. C'est cette version amincie qui va cependant se perpétuer.

Transmission de la mémoire et amnésie partielle

Le premier historique de la garde républicaine n'est rédigé qu'en 1857 sur ordre de l'inspecteur général ${ }^{93}$. Un des objectifs de ce journal dit des marches et opérations est donc de mettre en exergue le caractère militaire de la garde républicaine et de ses devancières. Cet historique va donc occulter les aspects policiers de son action. Il vise aussi à souder les gardes autour d'une mémoire commune. La Notice historique de Cochet de Savigny avait la même ambition pour l'ensemble de l'arme. Il est donc exclu d'évoquer les divisions passées. Par ailleurs, ses auteurs n'utilisent pas de témoignage de gardes qui ont participé aux événements. En effet, ils se contentent de reprendre textuellement le passage de la notice de Cochet de Savigny concernant la garde. Cette citation montre du reste le statut quasi officiel des écrits du rédacteur du Journal de la gendarmerie. Les auteurs y ajoutent seulement un témoignage de satisfaction de Gastu au corps et une lettre, du 10 février 1852, du commandant de la garde républicaine au général commandant la brigade de réserve. Cette lettre rédigée "en exécution d'une circulaire du ministre de la Guerre du 4 [février] ", « relative à la position que ce corps a occupé lors des événements de décembre » n'est qu'un résumé du rapport de Gastu du 10 décembre 1851. Ces documents officiels ne font l'objet d'aucun commentaire et ne sont accompagnés d'aucun récit complémentaire. Cet historique ne fait que rappeler la présence de la garde parmi les forces qui ont participé au rétablissement de l'ordre.

François Cudet, lieutenant à la garde républicaine, affirme en 1887 : «Les événements du 2 décembre n'intéressent pas le corps. La garde républicaine n'eut aucune mission à remplir. Commandée par le colonel Gastu, elle était paisiblement sous les armes dans la cour de la caserne de la Cité, c'est à dire aux environs de la Préfecture de police " ${ }^{94}$. On peut en douter, d'autant plus que la caserne de la Cité n'est achevée qu'en 1867! Un autre historique du corps, écrit en $1906{ }^{95}$, répète que : «Le rôle de ce corps, lors du coup d'État fut négatif : c'est peut-être la seule commotion parisienne où il n'ait pris aucune part $"{ }^{96}$.

D'autres motivations animent cependant ces auteurs. Il est en effet mal venu, sous la Troisième République, d'évoquer des actions contre des républicains, d'autant que ces historiques insistent sur la vocation de la garde à défendre, dès sa formation, la République. Notons que les actions du corps lors des journées de juin 1848 sont, quant à elles, très détaillées. Ils occultent ainsi, eux aussi, à une époque où la garde républicaine comme la gendarmerie veulent réaffirmer leur caractère militaire, les aspects policiers de l'action de la garde au 2 décembre.

Cette amnésie des militaires, cet embarras à dire, se perpétue tout au long du $\mathrm{XX}^{\mathrm{e}}$ siècle. Le général Louis Larrieu ${ }^{97}$ dans son Histoire de la gendarmerie, pourtant fort documentée, n'évoque pas la garde républicaine lorsqu'il traite des événements de décembre 1851. Il fait en revanche un éloge des gendarmes des départements pour leur lutte contre les résistances au coup d'État.

La crainte d'avoir des défections parmi les gardes ne s'est pas donc réalisée. Les autorités ont estimé en définitive que la garde républicaine ne présentait pas plus de risques d'insubordination qu'un autre régiment de Paris. Sa position stratégique dans le 
dispositif en témoigne. En décembre 1851, la physionomie de la garde, est bien différente de celle de juin 1848. La garde est désormais composée d'anciens gardes municipaux dont il est fort probable, à l'image de Saint-Arnaud, qu'ils ont au « cœur la rancune de Février $"{ }^{98}$. Les radicaux ne sont plus qu'une poignée. Les circonstances des combats diffèrent également de celles de juin. L'essentiel de la garde n'est pas en contact direct avec les émeutiers et donc avec d'éventuels anciens camarades. De plus, l'effet de corps joue dans une troupe solidement encadrée. L'autorité des officiers est respectée. L'idée que la garde républicaine est un acteur politique a vécu. Pour l'ensemble de ses militaires, ils ne s'agissaient pas non plus de mettre en danger une carrière. Enfin, l'ambiguïté de l'image de Louis-Napoléon dans l'opinion et l'ambivalence du coup d'État - restauration du suffrage universel et coup de force antiparlementaire - peuvent aussi aider à comprendre le maintien de la cohésion de la garde. Ce sont toutes ces mêmes raisons qui expliquent le ralliement massif de l'armée au coup d'État.

L'engagement de la garde républicaine dans les combats est minime. Ses missions sont surtout policières. Cet état de fait pose des problèmes aux militaires chargés de consigner la mémoire du corps. L'analyse de ces discours - et de ces silences - illustre les schémas et les enjeux politiques de la construction d'une mémoire collective. Répondant à des enjeux identitaires propres au corps, ils occultent les missions de police peu prestigieuses. Ils transforment le coup d'État en un non-événement de l'histoire de la garde. Sa faible visibilité lors des journées de décembre 1851 favorise la genèse de cette mémoire sélective. La participation de la « garde de la République » à la répression est ainsi minorée pendant le Second Empire, puis, sous la République, tout simplement niée. Le contexte politique-c'est-à-dire républicain-va, lui, ensuite, contribuer à la perpétuation de cette amnésie, ou plus exactement de ce refoulement.

\section{NOTES}

1.. Pierre MAYER, Histoire du deux décembre, avec des documents inédits et pièces justificatives, Paris, Ledoyen, 1852, in-18, pp. 71-72. Adolphe GRANIER de CASSAGNAC évoque également l'anecdote dans Récit complet et authentique des évènements de décembre 1851 à Paris et dans les départements, Paris, Impr. de Lange-Lévy, 1851, in-8: p. 11, mais date le vol du 24 février et attribue le numéro 478 au sabre. On trouve la trace de ce méfait dans la déclaration de Kerkan, voir Arch. nat. (Archives nationales) F ${ }^{\circ} 91173$, dommages de février 1848. Anciens militaires de la garde municipale demandant des indemnités. Dossiers individuels.

2.. Éric ALARY, L'Histoire de la gendarmerie. De la renaissance au troisième millénaire, préface de René Raymond, Paris, 2000, Éditions Calmann-Lévy, 286 p., traite le coup d'État en 4 lignes (p. 80-81) et n'évoque pas la garde républicaine.

3.. On reprend ici, transposés au coup d'État, les problèmes soulevés par Rémi GOSSEZ dans « Notes sur la composition et l'attitude politique de la troupe », dans Bibliothèque de la révolution de 1848, Études, t. XVIII, La Roche-sur-Yon, 1955, pp. 77-110. 
4.. Un pamphlet de mai 1849 explique que la « garde [municipale] a été lâchement assassinée et chassée par les démagogues et leurs amis les repris de justice ; ses casernes ont été pillées et en partie incendiées. Leurs chevaux leur ont été volés par une horde de véritables brigands sortis on ne sait d'où ", dans Louis DURAT-LASALLE, Ce qui arriverait si... Aux vieux soldats pensionnaires de l'État, aux braves de l'Hôtel des Invalides et de la succursale, aux membres de la légion d'honneur, aux marins de l'État et du commerce, à la gendarmerie nationale, Paris, Impr. Wittersheim, 1849, in-16, p. 23. Les gardes municipaux menacés et pourchassés par la foule ont eu, en plus, nombre de leurs effets et pour certains tout leur mobilier détruits ou volés. Voir Arch. nat. $\mathrm{F}^{9}$ 1172-1176 dommages de février 1848. Anciens militaires de la garde municipale demandant des indemnités. Dossiers individuels.

5.. Lettre du ministre de l'Intérieur au préfet de police du 12 janvier 1852, Arch. nat. F9 1171,dommages de Février 1848. Militaires de la gendarmerie 1850-1852.

6. Commandant en chef des gardes nationales de la Seine et des troupes de la $1^{\text {ère }}$ division militaire.

7.. Charles-Émile de MAUPAS, Mémoires sur le Second Empire, Paris, Éditions E. Dentu, 1884-1885, in-8, t. I, p. 316.

8.. Lettre de Changarnier au ministre de la Guerre du 18 février 1850, SHAT (Service historique de l'Armée de Terre) dossier du général de division Gastu, 7 Yd 1354.

9.. Paul BELOUINO, Histoire d'un coup d'État (décembre 1851) d'après les documents authentiques, les pièces officielles et les renseignements intimes, Paris, Éditions Brunet, 1852, in-8 : pp. 200-201.

10.. Xavier DURRIEU, Le coup d'État de Louis Bonaparte. Histoire de la persécution de décembre, événements, prisons, casemates et pontons, Londres/Genève/New York, J. Thomas, 1852, in-32, p. 24 et Jean Baptiste Adolphe CHARRAS (colonel), Enquête sur le 2 décembre et les faits qui le suivent, Bruxelles, 1852, in-12, p. 33.

11.. Moniteur parisien du 7 décembre 1851. Cette information est reprise par : Edmond ALONNIER et Joseph DÉCEMBRE, Le coup d'État du 2 décembre 1851. Historique des événements qui ont précédé le coup d'État, physionomie de Paris, arrestations et barricades, types et portraits des principaux personnages, faits qui ont suivi la chute de la République, pièces et documents officiels, Paris, Décembre-Alonnier libraire-éditeur, 1868, in-18, p. 203 ; Victor SCHOELCHER, Le Deux décembre, les massacres dans Paris, Paris, Librairie de la bibliothèque démocratique, 1872, in-32, p. 126 ; Hippolyte MAGEN, Histoire du Second Empire, Paris, Editions M. Dreyfous, 1878, p. 120. Ce dernier place le fait le 5 décembre. 12.. Arch. nat. $F^{7}$ 2588-2595. Événements de décembre 1851. Décisions des commissions mixtes dans toutes la France, par ordre alphabétique. On a également consulté la liste générale des individus poursuivis dans la première division militaire à l'occasion de l'insurrection de décembre 1851, Arch. nat. (BB $\left.{ }^{22} 190\right)$ qui donne des indications sur les professions de ces individus.

13. Aux Arch. nat, demandes de grâces : Boiteux (BB $\left.{ }^{22} 159\right)$ et Élie (BB $\left.{ }^{30} 475\right)$. Au SHAT : dossiers des conseils de guerre et des commissions mixtes de la première division militaire : Boiteux (7J 37), Élie (7J 851), Beaumont (7J 269), Bourdenet (7J 263), Caillaud (7J 160), Jollivet (7J 920), Lacuve (7J 616-845), Luneau (7J 1494), Gervais (7J 1962), Pornin (7J 688-711). Renet (7J 726, dossier manquant) et Hilaire (7J 18, secrétaire du député Xavier Durrieu, dossier manquant car condamné par la commission de l'Ariège). Pour Bressy, ancien chirurgien des Montagnards condamné à Reims, cf. Gustave LAURENT, « Exposé des sources bibliographiques. Les événements de l'année 1848 à Reims et dans la Marne ", dans Le département de la Marne et la Révolution de 1848, Comité départemental 
marnais de célébration du centenaire de la Révolution de 1848, Châlons-sur-Marne, 1948, pp. 73-78.

14.. SHAT, 7J 263 Bourdenet. Rapport de Marquisan sur l'arrestation de Bourdenet adressé au colonel de la garde républicaine, du 6 janvier 1852.

15.. Eugène TENOT, Paris en décembre 1851. Étude historique sur le coup d'État, Paris, A. Le Chevalier, 1868, in-8, p. 219 et 232 ; Victor HUGO, Histoire d'un crime, déposition d'un témoin, dans le tome « Histoire » des CEuvres complètes, Paris, Robert Laffont, 1987, p. 344 ; H. MAGEN, ouv. cité, p. 106 ; A. CHARRAS, ouv. cité, p. 53 ; E. ALONNIER et J. DÉCEMBRE, ouv. cité, pp. 189-190.

16.. SHAT, J7 688-711 Pornin. Déposition de Julien Thuillier du 12 janvier 1852,. 17.. La proportion exacte de combattants de février dans l'effectif du corps en décembre 1851 sera déterminée dans ma thèse de doctorat: Des gendarmes à Paris. La garde républicaine et la garde de Paris 1848-1871, sous la direction de Jean-Noël LUC, Université Paris IV.

18.. SHAT, Dossier 7J 160 Caillaud et Victor HUGO, ouv. cité, p. 392 et déposition de Caillaud, p. 512.

19.. SHAT, 7 J 37 Boiteux, Procès-verbal d'interrogatoire par le commissaire de police du 7 décembre 1851.

20.. SHAT, $G^{9} 1$, Rapport du général de la Rüe, inspecteur général de la garde de Paris, du 30 octobre 1853.

21.. MARQUISAN (chef d'escadron major), PEIFFER (capitaine), VESCO (lieutenant), Garde de Paris. Journal historique du corps, manuscrit, p. 175-178, Bibliothèque Historique de la Ville de Paris, C. P. 5219.

22.. Il est vrai que les cas d'insubordination sont très rares.

23.. Albert DU CASSE (baron), Histoire anecdotique du Second Empire, par un ancien fonctionnaire, Paris, E. Dentu, 1887, in-8, pp. 85-86.

24.. Arrêté ministériel du 4 août 1849.

25.. Le Journal de la gendarmerie, publié et rédigé par Pierre-Claude-Melchior COCHET de SAVIGNY (chef d'escadron), Paris, Éditions Léautey et Lecointe, in-8 ${ }^{\circ}$, numéro du 16 février 1850.

26.. Journal de la gendarmerie, ouv. cité, numéro du 9 août 1850.

27.. Journal de la gendarmerie, ouv. cité, numéro du 21 novembre 1851.

28.. SHAT, 7J 37 Boiteux, Lettre de Boiteux depuis la casemate 20 du fort d'Ivry, s.d.

29.. Arch. nat. $\mathrm{BB}^{30} 394$, Lettre du parquet du procureur de la République au procureur général de la Cour d'appel d'Angers, du 17 octobre 1851, affaires politiques, dossier $\mathrm{P}$ 381.

30.. Arch. nat. B II $1134^{\mathrm{C}}$, Votes de la garde républicaine lors du plébiscite de 1851.

31.. Victor HUGO, ouv. cité, p. 550.

32.. Dans tout le pays, la consultation de l'armée donne pour résultats 37359 non, 3626 abstentions, pour 303290 oui. Henri GUILLEMIN, Le coup du 2 décembre, Paris, Éditions Gallimard, collection La suite des temps, 1951, in-8º p. 419.

33.. Voir SHAT, $\mathrm{F}^{1} 32$ et $\mathrm{F}^{1} 33$, Notamment Changarnier. Lettres de Changarnier au général Neumayer du 22 mars et du 29 avril 1850.

34.. SHAT, $\mathrm{F}^{1}$ 51. Événements du 2 décembre. Armée de Paris. Général Magnan, commandant en chef. Dispositions en cas de combat.

35.. On peut y lire en effet des corrections, quant au dispositif de la garde républicaine, tenant compte de la réorganisation de sa cavalerie en quatre escadrons datant de décembre 1852. 
36.. « On m'avait remis, à la Préfecture de police, le plan ou plus exactement les plans qu'avait préparés $M$. Carlier, mon prédécesseur, en vue d'un coup d'État possible et auquel il avait dû participer vers le milieu de septembre. ", C.-É. de MAUPAS, ouv. cité, t. I, p. 207.

37.. SHAT, $\mathrm{F}^{1}$ 51. Lettre de Gastu au général commandant la $1^{\text {ère }}$ division militaire du 3 décembre 1851.

38.. Arch. préf. pol. Paris (Archives de la préfecture de police de Paris), AA 433 Lettre du préfet de police à la commission municipale du 17 décembre 1851 .

39.. C.-É. de MAUPAS, ouv. cité, t. I, p. 316.

40.. SHAT, Xf 135 bis, Gendarmerie mobile. $1^{\mathrm{er}}$ bataillon. État nominatif de MM. les officiers, les sous-officiers et gendarmes qui ont contribué par des combats au rétablissement de l'ordre pendant les journées des 2, 3, 4 et 5 décembre 1851, fait le 11 février 1852 par le chef d'escadron commandant, Saucerotte et SHAT, Xf 135, Gendarmerie mobile. $2^{\mathrm{e}}$ bataillon. État nominatif des militaires du bataillon qui ont concouru à maintenir ou rétablir l'ordre pendant les événements de décembre 1851 , avec indication du lieu où ils se trouvaient les $2,3,4,5$ et 6 du même mois, fait le 11 février 1852 par le chef d'escadron commandant.

41.. SHAT, $\mathrm{F}^{1}$ 52, Rapport du colonel Gastu sur l'action de la garde républicaine pendant les événement de décembre adressé au général Dulac, commandant la brigade de réserve, du 10 décembre 1851.

42.. Signalons ici que les témoignages des contemporains, dont l'Histoire d'un crime de Victor Hugo si fréquemment utilisé, sont souvent approximatifs. Ce dernier évoque notamment des gendarmes mobiles lorsqu'il s'agit de gardes républicains et inversement. La confusion entre les deux uniformes ne constitue pas une explication satisfaisante. Les dissemblances sont trop nombreuses et tous les contemporains savent les distinguer. Hugo utilise également des sources sans les vérifier.

43.. SHAT, $\mathrm{F}^{1}$ 52, Rapport d colonel Gastu.

44.. Victor HUGO, ouv. cité, p. 164. Il y aurait eu trente hommes selon A. GRANIER de CASSAGNAC, ouv. cité, p. 6 ; quarante d'après E. ALONNIER et J. DÉCEMBRE, ouv. cité, p. 100 ; quarante-cinq selon Taxile DELORD, Histoire du Second Empire 1848-1869, Paris, Germer Baillière, 1869, in-8 ${ }^{\circ}$, t. I, p. 293.

45.. C.-É. de MAUPAS, ouv. cité, p. 316.

46.. Victor HUGO, ouv. cité, p. 326.

47.. Polytechnicien qui avait fui en Amérique après avril 1834, il devient correspondant du National à New York puis administrateur de ce même journal après la révolution de 1848 et enfin préfet de la Marne sous Cavaignac.

48.. Victor HUGO, ouv. cité, pp. 534-535.

49.. SHAT, $\mathrm{F}^{1}$ 52, Rapport du colonel Gastu, ouv. cité

50.. Victor HUGO, ouv. cité, p. 195 ; Eugène TENOT, ouv. cité, p. 169 ; C.-É. de MAUPAS, ouv. cité, p. 389 ; Hyacinthe-Hippolyte de MAUDUIT (capitaine), Révolution militaire du 2 décembre 1851, Paris, A. Delahays, 1852, in-12, p. 145 ; et Charlemagne-Émile de MAUPAS, Rapport du préfet de police sur les évènements de décembre 1851, Paris, Imprimerie de Ch. Lahure, 9 rue de Vaugirard, 1853, p. 8.

51.. Victor HUGO ouv. cité, p. 318 et 481.

52.. Arch. préf. pol. Paris, AA 433 « Fournitures de vivres faites d'urgence aux officiers de la garde républicaine et soldats de service au Palais de Justice, à la Préfecture de police et à la prison Mazas pendant la journée du 4 décembre 1851: 4107 f 65 c », Brouillon de caisse. Dépenses du 5 décembre 1851. 
53.. Eugène TENOT, ouv. cité, p. 166.

54.. SHAT, $\mathrm{F}^{1}$ 52, Rapport du colonel Gastu, et C.-É. de MAUPAS, Mémoires..., ouv. cité, p. 433.

55.. SHAT, $\mathrm{F}^{1}$ 52, Rapport du colonel Gastu.

56.. Idem.

57.. C.-É. de MAUPAS, Mémoires..., ouv. cité, p. 423.

58.. E. ALONNIER et J. DÉCEMBRE, ouv. cité, p. 163 ; P. BELOUINO, ouv. cité, p. 165 ; SHAT, $\mathrm{F}^{1} 52$, rapport du colonel Gastu.

59.. SHAT, 7J 109 Lancien, il s'agit d'Isidore Jules Lancien, âgé de 17 ans, commis en épicerie, né à Sors (Seine inférieure). Procès-verbal du garde Jacquillon, s.d. et procèsverbaux d'interrogatoire des 3 et 14 décembre 1851 de Lancien.

60.. Lettre du général en chef Magnan au ministre de la Guerre du 3 décembre 1851, $\mathrm{F}^{1}$ 51, SHAT. Cité également par H. de MAUDUIT, ouv. cité, p. 154 et A. GRANIER de CASSAGNAC, ouv. cité, p. 32.

61.. Victor HUGO, ouv. cité, p. 343.

62.. Ibidem, p. 331.

63. Rapport de Gastu, ouv. cité

64.. C.-É. de MAUPAS, Rapport..., ouv. cité, p. 18.

65.. C.-É. de MAUPAS, Mémoires..., ouv. cité, p. 484.

66.. H. de MAUDUIT, ouv. cité, p. 239. Mauduit a eu accès au rapport du colonel Gastu pour l'élaboration de son récit. Voir aussi le témoignage de Charles Hugo, fils de Victor emprisonné à la conciergerie, Victor HUGO, ouv. cité, p. 547 et à propos de la seconde fusillade, E. ALONNIER et J. DÉCEMBRE, ouv. cité, p. 202.

67. SHAT, 7J 263 Bourdenet, Jean Marie Le Floch, âgé de 31 ans, né à Pleilot (Côte-duNord), demeure $n^{\circ} 2$ rue de la Vacherie. Il est arrêté sur la place du Palais de Justice, au coin de la rue du quai aux Fleurs. Blessé, il décède le 5 à l'Hôtel Dieu.

68. Nous signalons que la garde n'a eu aucun tué lors des événements de décembre.

69.. SHAT, 7J 263 Bourdenet.

70.. SHAT, $\mathrm{F}^{1} 51$, Lettre du général Renault au général commandant en chef l'armée de Paris du 4 décembre 1851, et Moniteur universel du 5 décembre 1851.

71.. Xavier DURRIEU, ouv. cité, p. 97.

72.. Victor HUGO, ouv. cité, p. 392 et E. ALONNIER et J. DÉCEMBRE, ouv. cité, note 1, p. 203.

73.. H. de MAUDUIT, ouv. cité, p. 240.

74.. H. de MAUDUIT, ibidem, p. 238 et Victor SCHOELCHER, ouv. cité, pp. 111-113.

75.. SHAT, 7J 263 Bourdenet, Lettre de Gastu au juge d'instruction du 8 décembre.

76.. Victor HUGO, ouv. cité, p. 335.

77.. Ibidem, déposition de Caillaud, p. 512.

78. Ibidem, déposition de Charles Hugo, p. 547 ; E. ALONNIER et J. DÉCEMBRE, ouv. cité, p. 202.

79.. C.-É. MAUPAS, Mémoires..., ouv. cité, p. 488.

80.. C.-É. de MAUPAS, Rapport..., ouv. cité, p. 18.

81.. Eugène TENOT, ouv. cité, p. 238.

82.. Rapport du général Magnan au ministre de la Guerre sur les événements parisiens de décembre, du 9 décembre 1851, dans Moniteur universel du 12 décembre 1851.

83. Il ajoute qu' « aucun engagement sérieux n'a eu lieu dans la Cité, mais partout où des agressions partielles se sont manifestées, elles ont été repoussées vigoureusement », SHAT, $\mathrm{F}^{1}$ 52, rapport du colonel Gastu. 
84.. «Ce rapport ne m'apprend rien et me confirme dans l'opinion que M. le colonel Gastu avec 2000 hommes d'élite s'est laissé enfermé dans la Préfecture ».

85.. Louis SAUREL (capitaine), La Gendarmerie dans la société de la Seconde République et du Second Empire, thèse de doctorat d'histoire, Université de la Sorbonne, 1957, t. III, p. 773.

86.. SHAT, $\mathrm{F}^{1} 52$, Rapport du colonel Gastu.

87.. P.-C.-M. COCHET DE SAVIGNY, ouv. cité, p. 19.

88.. Journal de la gendarmerie, ouv. cité, numéro du 21 décembre 1851.

89.. Décret du 24 décembre 1851 qui ouvre un crédit pour les indemnités à accorder aux particuliers et aux militaires de la garde municipale qui ont souffert des dommages matériels, par suite des événements de février et juin 1848, Mémorial de la gendarmerie, Paris, Éditions Léautey, 1855, t. IV, p. 394. La garde municipale est comprise dans ce crédit pour une somme de $194230 \mathrm{f}$.

90.. P.-C.-M. COCHET de SAVIGNY (chef d'escadron), Notice historique sur la révolution du mois de décembre 1851. Coopération de la gendarmerie dans la répression des troubles, actes remarquables de courage et de dévouement, pertes et récompenses, Paris, Éditions Léautey, 1852 , in- $8^{\circ}, 54 \mathrm{p}$.

91.. Ibidem, p. 19.

92.. Ibidem.

93.. Sur cet historique et ses enjeux, cf. l'auteur, « Miroir ! Ô beau miroir ! Le premier historique de la garde républicaine », dans Figures de gendarmes, Jean-Noël LUC [dir.], Sociétés et représentations, $\mathrm{n}^{\circ} 16$, septembre 2003, pp. 281-294.

94.. François CUDET, Histoire des corps de troupes qui ont été spécialement chargés du service de la ville de Paris depuis son origine jusqu'à nos jours, Paris, L. Pillet, 1887, p. 104.

95.. Sur cet historique et les conditions de la construction identitaire du corps, voir Marie-Berthe SERVIER, « L'historique de la garde républicaine. Une mémoire officielle conçue à la Belle Époque ", dans Jean-Noël LUC [dir.], Gendarmerie, État et société au XIX ${ }^{e}$ siècle, Paris, Publications de la Sorbonne, 2002, pp. 117-128.

96.. CLEMENT (capitaine), DIEZ (lieutenant), MICHELIN (chef d'escadron) (comité de rédaction), Historique de la légion de la Garde républicaine. tome I : Des origines à 1871, Paris, Garde républicaine, Presse régimentaire, 1908, p. 139.

97. Louis LARRRIEU (général), Histoire de la maréchaussée et de la gendarmerie, préface du général Georges Philippot, Ivry-sur-Seine/ Maisons-Alfort, Phénix éditions/ S.H.G.N., $2002,729 \mathrm{p}$.

98. Victor HUGO, ouv. cité, p. 165. H. de MAUDUIT parle lui de « revanche », ouv. cité, p. 187.

\section{RÉSUMÉS}

L'historiographie de la gendarmerie et de la garde républicaine présente cette dernière comme peu ou pas impliquée dans les événements du coup d'État. En revanche, de nombreuses sources et les témoignages des contemporains, quelles que soient leurs opinions politiques, font état d'une réelle participation des gardes républicains à la répression. Pour comprendre l'état d'esprit de cette troupe à la veille du 2 décembre, il convient de revenir sur son évolution depuis sa 
formation en 1848. L'étude détaillée de son action lors des journées parisiennes de décembre 1851 permet d'expliquer pourquoi et comment son rôle fut minoré.

The " guard of the Republic " and the $\mathbf{1 8 5 1}$ coup. Historiography about the gendarmerie and the Republican guard denies any serious involvement of the latter in the December events. On the other hand, several sources and testimonies of the contemporaries - whatever their political opinions - show a real participation of republican guards in the repression. To understand the frame of mind of these troops on the eve of the coup, it is necessary to detail its evolution since his formation in 1848. The precise study of his action during the Parisian days of December 1851 helps to explain why and how its part was played down.

\section{AUTEUR}

\section{FABIEN CARDONI}

Doctorant à l'Université Paris IV-Sorbonne et chargé de recherches au Service historique de la Gendarmerie nationale 